\title{
SCIDiC
}

\author{
International Journal of Dentistry and Oral Science (IJDOS) \\ ISSN: $2377-8075$
}

\section{Surgical Treatment Modalities in the Management of Temporomandibular Joint Disorders}

Research Article

\section{P. Santhosh Kumar ${ }^{1 *}$ K. Murugesan ${ }^{2}$, Nashwah Hinaz ${ }^{3}$}

${ }^{1}$ Professor, Department of Oral and Maxillofacial Surgery, Saveetha Dental College and Hospitals, Saveetha Institute of Medical and Technical Sciences (SIMATS), Saveetha University, Chennai 600077, Tamil Nadu, India.

${ }^{2}$ Professor and Head of the Department, Department of Oral and Maxillofacial Surgery, Saveetha Dental College and Hospitals, Saveetha Institute of Medical and Technical Sciences (SIMATS), Saveetha University, Chennai 600077, Tamil Nadu, India.

${ }^{3}$ Department of Oral and Maxillofacial Surgery, Saveetha Dental College and Hospitals, Saveetha Institute of Medical and Technical Sciences (SIMATS), Saveetha University, Chennai 600077, Tamil Nadu, India.

\section{Abstract}

Objectives: Temporomandibular joint disorders (TMD) are diseases that affect the temporomandibular joint and the supporting structures. The goal of treatment for temporomandibular disorders is the elimination of pain and return to its normal function. This aim of this study was to assess the various surgical treatment modalities employed in the management of Temporomandibular joint disorders and to evaluate the oral health related quality of life post-surgery in patients with temporomandibular disorders visiting saveetha dental college.

Methods: This retrospective study included data of 53 subjects who reported to Saveetha Dental College and diagnosed with TMD and surgically operated during June 2019 - May 2021. After assessment in the university patient data registry, consecutive case records of 30 patients who were diagnosed with Temporomandibular joint disorders [Disc-condyle complex] and underwent surgical therapy was included in the study; and consecutive case records of 23 patients with TMJ ankylosis who underwent surgical treatment were also included in the study. Descriptive (Frequency, percentage, mean and standard deviation) and Inferential statistics (chi square test, paired ' $t$ ' test) were employed Using SPSS software. Level of significance was set at $\mathrm{p}<0.05$ and results obtained.

Results: The age group of 31-40 years underwent most of the surgical treatment for TMD (31\%). Male patients predominantly underwent surgery for TMD (55.17\%). Among patients undergoing surgical treatment for TMD, TMJ subluxation/ dislocation was present in $44.83 \%$ of patients followed by disc displacement without reduction (41.38\%). $41.38 \%$ of patients were treated by High condylectomy and discopexy. The association between age and gender of the patients, type of temporomandibular joint disorder with the type of surgical treatment done for TMD was evaluated and the results were statistically significant $(\mathrm{p}<0.05)$. The age group of 11-20 years presented with higher incidence of TMJ ankylosis (36.36\%). Males showed higher incidence of TMJ ankylosis with $63.64 \%$. Sawhney's type II (45.45\%) was predominantly present among the TMJ Ankylosis patients. Right side of TMJ showed higher incidence of TMJ ankylosis (45.45\%). Interpositional arthroplasty was the predominant surgical treatment done for TMJ ankylosis patients (86.36\%). Dermis fat graft was commonly used in $63.64 \%$ patients as an interpositional material. The association between age and gender of the patients, site of involvement of TMJ ankylosis with Sawhney's classification of TMJ ankylosis was evaluated and the results were statistically significant $(\mathrm{p}<0.05)$.

Conclusion: It can be concluded from our study that TMJ disorders are now becoming common among the younger population especially the males. High condylectomy and discopexy was the commonest procedure done successfully for patients with disc-condyle disorders. High condylectomy with eminectomy was predominantly done for TMJ subluxation/dislocation. Interpositional arthroplasty with dermis fat graft was the predominant procedure done for treating TMJ ankylosis patients and the results were stable. This study highlights the role of various surgical treatment modalities to treat TMD and the high-risk group for developing temporomandibular joint disorders. Early diagnosis and adequate surgical intervention would minimize the deformity in TMJ ankylosis patients. Thus, surgical treatment modalities can be advised for indicated cases in TMDs, where conservative treatment modalities cannot offer proper cure for the patients. In certain cases of TMD, surgical modality may be the first and only option for the management. When performed at prompt time, surgical modalities can improve form, function and aesthetics of the patients thereby improving the overall outcome and general and oral health related quality of life of the patients.

Keywords: Temporomandibular Joint Disorder; Surgical Treatment; Tmj Ankylosis; Sawhney's Classification; Tmj Dislocation; Condylectomy; Tmj Internal Derangement; Pain; Quality Of Life; Mouth Opening.

*Corresponding Author:

Dr. M. P. Santhosh Kumar M.D.S.

Professor, Department of Oral and Maxillofacial Surgery, Saveetha Dental College and Hospitals, Saveetha Institute of Medical and Technical Sciences (SIMATS), Saveetha University, 162, Poonamallee High Road, Velappanchavadi, Chennai 600077, Tamil Nadu, India.

Tel: 9994892022

E-mail: santhoshsurgeon@gmail.com

Received: July 30, 2021

Accepted: August 11, 2021

Published: September 03, 202

Citation: M. P. Santhosh Kumar, K. Murugesan, Nashwah Hinaz. Surgical Treatment Modalities in the Management of Temporomandibular Joint Disorders. Int J Dentistry Oral Sci. 2021;8(9):4168-4179. doi: http://dx.doi.org/10.19070/2377-8075-21000852

Copyright: M. P. Santhosh Kumar M.D.S. 2021. This is an open-access article distributed under the terms of the Creative Commons Attribution License, which permits unrestricted use, distribution and reproduction in any medium, provided the original author and source are credited. 


\section{Introduction}

Temporomandibular joint disorders (TMD) have an adverse effect on jaw function so that patients may present with limited mouth opening or difficulty chewing because of pain and locking in the temporomandibular joint (TMJ) [1]. Currently, temporomandibular disorders (TMD) refer to the causes responsible for the impaired function of the temporomandibular joints (TMJ) and the associated neuro-muscular system, which may provoke TMD-related pain [2]. The term TMD is not a diagnosis but a broad term that contains a number of disease entities, such as pain in masticatory muscles and temporomandibular joints, headache, disturbances in jaw movements and sounds in joints while opening and closing the mouth. The causes of these diseases/ symptoms are numerous and include trauma, systemic, iatrogenic, occlusal and mental health disorders [3-6]. While most patients recover with simple measures such as jaw rest and soft diet, others require professional care that may involve any combination of occlusal splint therapy, physiotherapy, medications and sometimes require surgical intervention to manage TMD as well [7].

\section{Temporomandibular Joint Disorders}

Temporomandibular joint disorders can be classified as follows: [8]

A. Myofascial pain and dysfunction

- Myositis

- $\quad$ Fibromyalgia

- Neuropathic pain

- $\quad$ Chronic pain syndrome

B. TMJ functional derangement

- Internal derangement - disc displacement

- Hypermobility disorders - dislocation

- Hypomobility disorders - ankylosis, post-traumatic

C. TMJ degenerative/inflammatory joint disease

- Osteoarthrosis/arthritis

- Rheumatoid arthritis

- $\quad$ Psoriatic arthritis

- Juvenile arthritis

TMD management strategies include the following: [8]

1. Explanation and reassurance

- TMD is not life-threatening

- TMD is not a Cancer

- TMD can become a chronic condition

- $\quad$ TMD can be managed

2. Education and self-care

- $\quad$ Soft diet

- Jaw rest (especially during long dental appointments)

- $\quad$ Avoid extreme jaw movements (i.e yawning)

- Topical heat (e.g. wheat packs)

- Protect face and jaws from cold weather

- $\quad$ Avoid stress and anxiety

3. Medications

- Antiinflammatories

- Anxiolytics

- $\quad$ Muscle relaxants

- Antidepressants

4. Jaw physiotherapy

- Massage and stretching

- $\quad$ Dry needling

- TENS - transcutaneous electrical nerve stimulation
- $\quad$ Pulsed ultrasound therapy

5. Occlusal appliance therapy

6. Behavioural therapy

- $\quad$ Lifestyle counselling

- Relaxation therapy

- Hypnosis

- $\quad$ Biofeedback

7. Psychotherapy

8. Other

- Acupuncture

- Botox injections

- Chiropractic manipulation

9. TMJ surgery

- $\quad$ Closed procedures like TMJ arthrocentesis and TMJ arthroscopy

- $\quad$ Open procedures like TMJ arthrotomy/arthroplasty and TMJ joint replacements.

The three most common temporomandibular disorders are myofascial pain and dysfunction, internal derangement and osteoarthrosis. Only certain patients who are vulnerable to temporomandibular disorders will develop pain and dysfunction following an exacerbating event such as acute physical or psychological trauma, which suggests perhaps an underlying genetic predisposition to TMD $[7,8]$. It is seen that only when the compensatory capabilities of the masticatory and the neuromuscular system are overstretched, dysfunction occurs resulting in clinical symptoms and manifests as pain, severe clicking, or limited mobility of the mandible, forcing the patient to seek help.

Treatment for TMD's usually starts with a conservative approach; if not responsive it is followed by minimally invasive and open surgical procedures. According to the literature, 5-10 per cent of all patients undergoing treatment for temporomandibular disorders require surgical intervention. There are a wide range of surgical procedures for TMD ranging from temporomandibular joint arthrocentesis and arthroscopy to the more complex open joint surgical procedures, referred to as arthrotomy. The type of surgical procedure is chosen based on the specific condition of the patient and the surgeon's expertise $[9,10]$.

TMJ surgery has a place in the treatment armamentarium of temporomandibular disorders. It has a major role in restoring and repairing damaged tissue or remove tissue that cannot be salvaged; and surgery is also used to promote healing of tissues by replacing missing tissues with grafts. While surgery is often considered as a last option for TMD, there are instances where surgery is the definitive and sometimes the only treatment option such as in cases of TMJ ankyloses, and tumours [11, 12].

Symptomatic history of TMD, Clinical features of intolerable joint specific pain and joint dysfunction, and Joint pathology in radiographs are the key criteria for TMJ surgical intervention. Specific indications for TMJ surgery include chronic severe limited mouth opening and gross mechanical interferences such as painful clicking, locking and crepitus that fail to respond to nonsurgical measures; and degenerative joint disease. The more localised the symptoms are to the TMJ, the more likely surgery will have a favourable outcome. It is critical to point out that surgery has no role in the management of patients with chronic pain syndrome or muscular problems that do not involve the joint itself $[13-16]$. 
Indications for TMJ surgery are as follows: [17]

1. Absolute indications

- Ankylosis - eg. Fibrous or osseous joint fusion

- Neoplasia - eg. Osteochondroma of the condyle

- Dislocation - ie. Recurrent or chronic

- Developmental disorders - eg. Condylar hyperplasia

2. Relative indications

- Internal derangement

- Osteoarthrosis

- Trauma

A. General indications

- Disorder not responding to non-surgical therapy

- Where the TMJ is the source of pain and dysfunction

a) Pain localised to the TMJ

b) Pain on functional loading and movement of the TMJ

c) Mechanical interference with TMJ function

B. Specific indications

- $\quad$ Chronic severe limited mouth opening

- Advanced degenerative joint disease with intolerable symptoms

of pain and joint dysfunction

- Confirmation of severe joint disease on CT scan or MRI

There are a myriad of TMJ surgical procedures which restore, repair or remove damaged or diseased joint tissues. TMJ surgery may be divided into two major groups: Closed

procedures such as TMJ arthrocentesis and TMJ arthroscopy, and open procedures such as TMJ arthroplasty and joint replacement surgery.

\section{Closed TMJ Surgical Procedures}

Temporomandibular joint arthrocentesis and arthroscopy are very effective in managing 'stuck' joints by the simple process of lubricating the superior joint space and introducing various medicaments such as steroids and hyaluronic acid; and allowing mobilization of the articular disc. While TMJ arthrocentesis is useful for cases of acute onset closed lock TMJ arthroscopy provides a more effective approach to the management of chronic $(>3$ months) or recalcitrant cases of closed lock.

Temporomandibular joint arthroscopy is a sophisticated version of arthrocentesis in which incredibly complex surgical procedures ranging from surgical debridement to disc repositioning and repair are done through miniature portholes by looking directly into the inside of the joint space for the pathology [18].

\section{Open TMJ surgical procedures}

Open TMJ surgery, referred to as arthrotomy, involves the surgical exposure of the TMJ via a preauricular incision. Arthrotomy is used when the joint itself is damaged through trauma, degenerative, or inflammatory disease. Arthrotomy permits a wide range of surgical procedures ranging from disc repair and repositioning (Discopexy) to discectomy, or removal of the entire disc that is beyond repair. Depending on the situation, tissue grafts may be required to replace joint components [disc and or condyle] which cannot be salvaged. In extreme cases of TMJ disease such as osteoarthritis, a complete condylectomy is performed and is reconstructed using a total TMJ prosthesis to maintain lower facial symmetry and to preserve the existing occlusion. TMJ prostheses especially with bilateral ones, only allow hinge action of opening and closing with limited lateral and protrusive excursions of the mandible. TMJ surgery should be followed up by physiotherapy and jaw exercises. The success of TMJ surgery depends on accurate diagnosis, appropriate case selection, skill and experience of the surgeon and post-operative rehabilitation program [19].

\section{Temporomandibular Joint Dislocation}

Temporomandibular joint (TMJ) dislocation can be debilitating for patients as it causes significant pain and anxiety for patients, and results in trauma to the joint capsule and ligaments. This dislocation may occur in one of four forms. The first form, subluxation, occurs when there are transient partial dislocations of the TMJ that are usually self-resolving. The second form, acute dislocation, usually occurs as a result of trauma or excessive mouth opening (e.g. during yawning or after prolonged dental procedures). Acute TMJ dislocation is managed via manual reduction, which may require sedation. This may be augmented with supportive bandages and soft diet. The third form, chronic dislocation, occurs when there is prolonged disarticulation of the TMJ, usually as a result of underlying laxity of the joint capsule. This may be secondary to age-related degeneration, an inherent connective tissue disorder (such as Ehler's Danlos Syndrome), or as an adverse effect of antipsychotic medication such as clonazepam. Chronic dislocation may be managed conservatively via positional devices, or surgically. The last form of TMJ dislocation is recurrent dislocation. This occurs when there are recurrent acute episodes of TMJ disarticulation due to either joint capsule/ ligament laxity, anatomical variation in the joint or dystonia of the lateral pterygoid muscle causing spasmodic antero-medial pull on the condylar head [20].

Factors contributing to recurrent TMJ dislocation include dystonia of the lateral pterygoid muscle and laxity in the meniscus and lateral TMJ ligament. Although usually described in middle-aged or elderly populations, recurrent TMJ dislocation can occur at all ages, including infants. Recurrent dislocations can further injure the disc, capsule and TMJ ligaments, contributing to tendency for progressive internal derangement and recurrence of dislocation. The risk factors, mechanisms, clinical features and management of each type of TMJ dislocation varies and the physician must be well aware of it [21].

Management of acute dislocation involves: Conventional technique-Hippocratic/Nelaton's Method; Wrist pivot technique; Extraoral technique and Gag reflex. Management of Chronic Recurrent Dislocation/subluxation involves: conservative, minimally invasive and surgical procedures. Conservative procedures are Physiotherapy, Intermaxillary fixation, Chin straps, Barton's bandage and Kinesio taping. Minimally invasive procedures are Injection of sclerosing agents, Autologous blood injection, Prolotherapy, and Botulinum toxin injection. Surgical procedures are Capsular tightening procedure [Capsulorrhaphy], Creation of mechanical obstacle [Dautrey's procedure, Glenotemporal osteotomy], Removal of mechanical obstacle [Eminectomy, Condylectomy] and Creation of new muscular balance [Temporalis scarification, Lateral pterygoid myotomy, Pterygoid dysjunction] [22].

Recurrent TMJ dislocation remains a management challenge for practitioners and treatment depends on the etiology. If a lax disc 
is implicated, disc plication with non-resorbable sutures or a mini orthodontic screw is advised. If the eminence form is implicated in the cause of recurrent dislocation, then eminectomy or eminoplasty procedures may be of benefit. If lateral pterygoid dystonia is causing recurrent dislocation, then lateral pterygoid myotomy or injection of botulinum toxin A into the muscle can help alleviate this problem. Patients are currently being successfully managed by eminectomy and disc plication. Eminectomy allows free movement of the condylar head, and avoids entrapment of the condylar anterior to the eminence, thereby avoiding the need for hospital presentation for reduction. Disc plication aims to promote synchronous movement of the condylar head and TMJ meniscus, thereby reducing ligamentous injury with hypermobility [23].

Injection of autologous blood into the superior joint space and lateral TMJ capsule has been extensively described in the literature, with a success rate of $80 \%$ at 16 months. Botulinum toxin injection of the lateral pterygoid muscle has also been reported to have a success of $80 \%$ at 6 months. Modified dextrose has been shown to be a promising new modality for the minimally invasive management of recurrent TMJ dislocation. A study of 45 patients reported a success of $91 \%$ at 18 months, which is higher than that reported by any other modality. The mainstay of surgical management of recurrent TMJ dislocation is eminectomy. However, disc plication has also shown excellent promise, with $100 \%$ success at 12 months in a group of 27 patients. Eminoplasty can also be effective, with several modifications described. The surgeon must consider the patients suitability for surgery and possible aetiology when deciding the best approach to management [23].

\section{Temporomandibular Joint Ankylosis}

Temporomandibular joint (TMJ) ankylosis is a refractory disease that restricts the movement of the mouth and its ability to open and can lead to subsequent maxillofacial deformities, speech impediments, difficulty chewing and narrow airways for patients who are at ages of active growth. The aetiology of TMJ ankylosis includes the fusion of the condyle and disc to the glenoid fossa by bone or fibrous tissue and is primarily caused by trauma, infection or secondary to treatment of a mandibular condylar fracture or other TMJ diseases [24].

TMJ ankylosis is classified by location (intra-articular or extraarticular), type of tissue involved (eg, bone, fibrous, or fibro-osseous), and extent of fusion (complete or incomplete). Trauma, radiotherapy, surgical excision of TMJ tumors, infection, and systemic disease can all result in mandibular hypomobility. Infection remains the most common cause of TMJ ankylosis in children. Local odontogenic, ear, and skin infections or osteomyelitis and systemic spread of osteomyelitis from the long bones are the most commonetiologies. Patients in the deciduous dentition, with intracapsular and/or comminuted fractures, are at the greatest risk for developing ankylosis. Radiotherapy produces fibrosis, scarring, and induration of the soft tissues surrounding the TMJs. Resection of a tumor involving the TMJ can result in fibrosis at the surgical site and limitation of jaw motion. Systemic autoimmune disorders, including ankylosing spondylitis, juvenile rheumatoid arthritis, and psoriasis can result in TMJ ankylosis. Temporomandibular joint (TMJ) ankylosis in children is one of the most difficult and complex problems to treat. TMJ ankylosis in the paediatric patient often leads to facial deformity, difficulty chewing and swallowing, and poor oral hygiene [24].

In 1986, Sawhney was the first to classify TMJ ankylosis into four types according to the anatomical relationships between the TMJ structure and the osseous fusion. According to this classification, ankylosis type $\mathrm{I}$ is caused by fibrous adhesion in and around the joints but with less bone fusion in the TMJ. Ankylosis type II has bone fusion around the lateral surface of the joint, but there is no additional bone fusion in the central region of the TMJ. Ankylosis type III can be caused by any injury to the condyle, whether treated or not, and is characterised by bone fusion between the mandibular ramus and glenoid fossa, and can even implicate the zygomatic arch. The condyle is medially displaced and may be reduced in size, but the surface of the joint maintains an identifiable anatomical structure. In ankylosis type IV, the entire joint is replaced by a bone fusion, which can even implicate the mandibular coronoid process, making it difficult to identify the anatomical structure of the TMJ [25].

Untreated TMJ ankylosis in children results in significant facial asymmetry because of the hypomobility and abnormal muscle function. The short ramus condyle unit restricts mid-face growth; secondary elongation and hypertrophy of the coronoid process occurs, further restricting jaw motion. The longer the duration of hypomobility, the more severe will be the muscle atrophy and facial asymmetry. The prognosis for a favourable outcome with treatment is inversely related to the number of years of ankylosis. Therefore, treatment of ankylosis should be done as soon as it is feasible to expect patient cooperation after the operation. Benefits from early operation include improved psychosocial development because of a more normal appearance, improved nutrition, improved oral hygiene and ability to obtain dental treatment. Children 3 years of age or older are considered candidates for ankylosis release. It is not necessary and actually contraindicated to wait for the completion of growth because the asymmetry will progress with time if the ankylosis is left untreated. Children 3 years of age and older can cooperate with physical therapy, provided the ankylosis release is successful and excessive force to mobilize the jaw is not necessary during the postoperative period. Failure under these circumstances is a failure of the operation and not a failure of patient cooperation [26].

Treatment objectives for TMJ ankylosis includes: Restoration of function (mouth opening), Restoration of aesthetics (posterior ramal height and facial symmetry) and to Prevent recurrence. Treatment options for adult ankylosis are: [27]

$$
\begin{aligned}
& \text { - Condylectomy } \\
& \text { - Gap arthroplasty } \\
& \text { - Interpositional arthroplasty: Temporalis muscle, fascia, } \\
& \text { fat, auricular cartilage, dermis } \\
& \text { - Reconstruction with costochondral graft, chondro-osse- } \\
& \text { ous iliac bone graft, alloplastic total joint prosthesis } \\
& \text { - } \quad \text { Aggressive postoperative physical therapy }
\end{aligned}
$$

Kaban's protocol for management of temporomandibular joint ankylosis (1990) includes: [27]

(a) Aggressive resection of the ankylotic segment

(b) Ipsilateral coronoidectomy

(c) Contralateral coronoidectomy when necessary 
(d) Lining the joint with temporalis fascia or cartilage

(e) Reconstruction of the ramus with a costochondral graft

(f) Rigid fixation of the graft

(g) Early mobilization and aggressive physiotherapy

Kaban's modified protocol for management of TMJ ankylosis in children (2009) includes: [28]

(a) Aggressive excision of fibrous and/or bony mass

(b) Coronoidectomy on affected side

(c) Coronoidectomy on opposite side if steps 1 and 2 do not result in MIO of $35 \mathrm{~mm}$ or to point of dislocation of opposite side

(d) Lining of joint with temporalis fascia or the native disk, if it can be salvaged

(e) Reconstruction of RCU with either DO or CCG and rigid fixation

(f) Early mobilization of jaw; if DO used to reconstruct RCU, mobilize day of surgery; if CCG used, early mobilization with minimal intermaxillary fixation (not $>10$ days)

(g) Aggressive physiotherapy

Gap arthroplasty, interpositional arthroplasty, and osteotomy across and excision of the ankylotic mass within the TMJ are the treatment options for TMJ ankylosis. Reconstruction of the ramus/condyle unit [RCU] with autogenous bone, such as a costochondral graft (CCG), fibula, clavicle, iliac crest, metatarsal head, or alloplastic material, have all been reported in the literature with varied results. Once the ankylotic mass has been removed, the joint must be lined with vascularized tissue. This acts as a barrier to excessive bone formation, fusing the RCU to the skull base. The insertion of soft tissue between the condyle and glenoid fossa is essential for the reduction of recurrence. A vascularized temporalis myofascial flap is desirable for lining the joint because the donor site is in the surgical field, the muscle and fascia are of adequate thickness, and its long-term viability has been demonstrated. The rib growth center is the costochondral junction and traditionally, the RCU was reconstructed using a CCG. However, overgrowth of CCGs occurred if an excessively large cartilaginous cap was used. Thus, including a CCG of only 1 to $2 \mathrm{~mm}$ of cartilage will prevent overgrowth. The benefits of a CCG include its growth potential, its biologic compatibility, and its capacity to remodel into a neo-condyle with time. Its major drawbacks are donor site morbidity and reported unpredictable growth. More recently lengthening of the residual ramus is performed using transport DO. Distraction osteogenesis (DO) has the advantage of eliminating donor site morbidity and allowing immediate mobilization of the jaw [29].

Limited range of motion and re-ankylosis [within 6 months after surgery] are the most frequently reported complications. This is most commonly caused by a failure to adequately excise the ankylotic mass, resulting in failure to achieve complete, passive opening (without the need for excessive force) in the operating room. If excessive force is necessary to open the jaw intraoperatively, even more force will be required postoperatively. Under these circumstances, physical therapy will be very painful, and the operation doomed to failure, regardless of the level of patient cooperation. Thus, children cannot or will not cooperate with physical therapy and hence will always have a poor outcome after ankylosis release. Re-ankylosis is most commonly caused by incomplete excision of the bony and/or fibrous mass, specifically on the medial aspect of the joint. In addition, many surgeons fail to appreciate the role of the ipsilateral and contralateral coronoid processes, along with the attached contracted temporalis muscles, in the limitation of motion in children with ankylosis. Increase in maximal mouth opening $(>35-40 \mathrm{~mm})$, reduction or absence of pain in TMJ, no deviation of jaw on mouth opening, lateral excursion movements are the factors that signify improvement in the quality of life, post ankylosis correction surgery [24].

Thus, the treatment for TMJ ankylosis aims to establish mandibular movement and TMJ functionality. To accomplish this, the bone fusion must be completely removed and the condyle or its substitute needs to maintain normal physiological function. A key component in the treatment of TMJ ankylosis is the approach taken to restore the original height of the mandibular ramus while preventing direct contact between the condylar process and the glenoid fossa.

This aim of this study was to assess the various surgical treatment modalities employed in the management of Temporomandibular joint disorders and to evaluate the oral health related quality of life post-surgery in patients with temporomandibular disorders.

\section{Materials and Methods}

\section{Study design and Study setting}

This retrospective study was conducted in the department of oral and maxillofacial surgery, Saveetha dental college and hospital, Saveetha University, Chennai, among patients who reported to our institution from June 2019 to May 2021 and were diagnosed with TMD. The study was initiated after approval from the institutional review board with the ethical approval number; SDC/ SIHEC/2020/DIASDATA/0619-0521.

\section{Study population and sampling}

Inclusion criteria for the study were adult dental patients with TMD. Exclusion criteria included immunocompromised patients, dental anomalies, systemic diseases with cognitive problems and speech problems, missing or incomplete data. After assessment in the university patient data registry, consecutive case records of 30 patients who were diagnosed with Temporomandibular joint disorders [Disc-condyle complex] and underwent surgical therapy was included in the study; and consecutive case records of $23 \mathrm{pa}-$ tients with TMJ ankylosis who underwent surgical treatment were also included in the study. Cross verification of data for errors was done with the help of an external examiner.

\section{Data collection and tabulation}

Data regarding patients having TMD were retrieved after analysing over 86000 case sheets. The following parameters were evaluated based on the dental records; age, gender, classification and types of TMD, site of involvement of TMD, and various types of surgical therapy provided for TMD. Chief complaints, medical and dental history, all the clinical manifestations of TMDs, treatment strategies and outcomes of the patients with the surgical therapy were examined from the data, collected and recorded. Patients diagnosed with TMD were further classified into disc displacement with reduction, disc displacement without reduction and TMJ dislocation/subluxation, degenerative disorder, myofascial pain and dysfunction syndrome (MPDS), and TMJ ankylosis. 
The clinical presentations of TMD were considered into several variables and the patients were classified according to the severity of the TMD for diagnosis, treatment and prognosis purposes. Quality of life after surgery was also assessed by evaluating the pre-operative and post-operative pain and mouth opening scores. Post-operatively the patients were recalled periodically and were reviewed and monitored during 1 st, 2nd, 3rd, 6th, 9th and 12th months and thereafter every 6 months. Data was entered in excel and was imported to SPSS. The variables were defined.

\section{Statistical Analysis}

The collected data was validated, tabulated and analysed with Statistical Package for Social Sciences for Windows, version 23.0(SPSS Inc., Chicago, IL, USA) and results were obtained. Descriptive analysis was used to describe the number of patients treated with surgical therapy based on age, gender, classification and types of TMD, and types of surgical therapy provided for the study population. Categorical variables were expressed in frequency and percentage; and continuous variables in mean and standard deviation. Chi-square test was used to test associations between categorical variables (age, gender, types of TMD, site of involvement of TMD and types of surgical therapy provided for treating temporomandibular joint disorders). Quality of life after surgery was assessed using pain scale - visual analogue scale [VAS], and mouth opening [both preoperative and post-operative] and the improvement in form and function was evaluated using paired 't'test. P value $<0.05$ was considered statistically significant.

\section{Results}

Our study consists of two parts: Surgical modalities performed for the patients with disc-condyle disorders [TMD] in the first part and for the patients with TMJ ankylosis [TMD] in the second part were evaluated.

In the first part of our study, 30 patients with TMD in the age range of 10-60 years who underwent surgical modality for the management of TMD were assessed. The age group of $31-40$ years underwent most of the surgical treatment for TMD $(31 \%)$ [Figure 1]. Male patients predominantly underwent surgery for TMD (55.17\%) than the females (44.83\%) [Figure 2].

Figure 3 depicts the type of temporomandibular joint disorders that occurred in patients requiring surgical treatment. TMJ subluxation/ dislocation was present in $44.83 \%$ patients undergoing surgical treatment followed by disc displacement without reduction $(41.38 \%)$ and disc displacement with reduction $(13.79 \%)$. Figure 4 shows the types of surgical treatment done for temporomandibular joint disorders. $41.38 \%$ of patients were treated by High condylectomy and discopexy and $27.59 \%$ of patients were treated with only high condylectomy. High condylectomy and eminectomy procedure was done for $17.24 \%$ of patients and discopexy procedure was performed for $13.79 \%$ patients.

The association between age of the participants and the type of surgical treatment done for TMD was evaluated and the results were statistically significant (Pearson's Chi square was done with $\mathrm{p}$

Figure 1. Bar graph showing age distribution of the patients undergoing surgical treatment for temporomandibular joint disorders. $\mathrm{X}$-axis represents the age group and $\mathrm{Y}$ axis represents the number of patients undergoing surgical treatment for temporomandibular joint disorders [TMD]. The age group of 31-40 years underwent most of the surgical treatment for TMD (31\%).

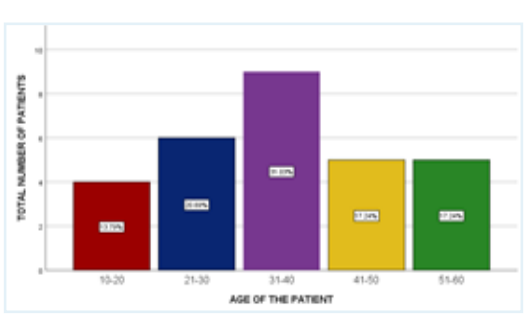

Figure 2. Bar graph showing gender distribution of the patients undergoing surgical treatment for temporomandibular joint disorders. $\mathrm{X}$ axis represents the gender and $\mathrm{Y}$ axis represents the number of patients undergoing surgical treatment for TMD. Male patients predominantly underwent surgery for $\operatorname{TMD}(55.17 \%)$.

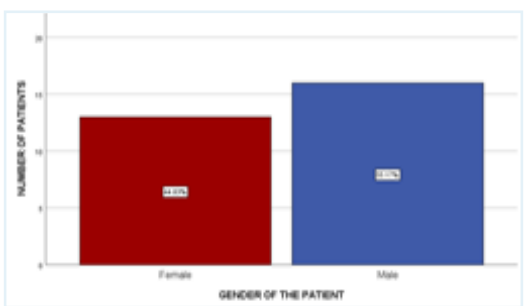

Figure 3. Bar graph showing distribution of type of temporomandibular joint disorders seen in patients requiring surgical treatment for TMD. $\mathrm{X}$ axis represents the type of TMD and $\mathrm{Y}$ axis represents the number of patients undergoing surgical treatment for TMD. $44.83 \%$ of patients with TMJ subluxation/ dislocation underwent surgical treatment for TMD followed by $41.38 \%$ of patients with disc displacement without reduction.

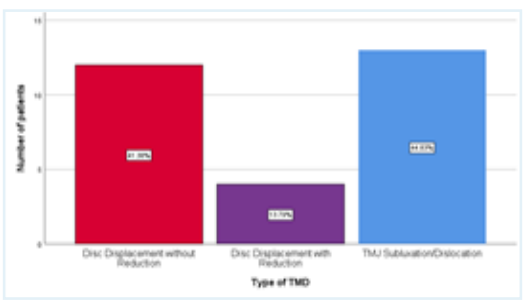


$=0.01(<0.05)$, hence statistically significant). High condylectomy and discopexy procedure was done predominantly $(24.14 \%)$ in the age group of 31-40 years. High condylectomy procedure was done predominantly $(13.79 \%)$ in the age group of $21-30$ years. High condylectomy and eminectomy procedure was done predominantly in 51-60 years age group (17.24\%). Discopexy procedure was done predominantly in $10-20$ years age group $(13.79 \%)$. Thus, the association between age of the participants and the type of surgical treatment done for TMD was statistically significant [Figure 5].

The association between gender of the participants and the type of surgical treatment done for TMD was evaluated and the results were statistically significant(Pearson's Chi square was done with $\mathrm{p}=0.044(<0.05)$, hence statistically significant). High condylectomy and discopexy procedure was done predominantly $(27.59 \%)$ in male participants. High condylectomy and eminectomy procedure was done predominantly $(17.24 \%)$ in female participants. Discopexy procedure was done only in male patients $(13.79 \%)$. Thus, the association between gender of the participants and the type of surgical treatment done for TMD was statistically significant [Figure 6].

The association between the type of temporomandibular joint disorder and the type of surgical treatment done for TMD was evaluated and the results were statistically significant(Pearson's Chi square was done with $\mathrm{p}=0.032(<0.05)$, hence statistically significant). High condylectomy with discopexy was the predomi- nant procedure done $(27.59 \%)$ for TMJ disc displacement without reduction, High condylectomy was predominantly done $(13.79 \%)$ for TMJ disc displacement with reduction, and High condylectomy with eminectomy was predominantly done (17.24\%) for TMJ subluxation/dislocation. Thus, the association between the type of temporomandibular joint disorder and the type of surgical treatment done for TMD was statistically significant [Figure 7].

In the second part of our study, 23 patients with TMJ Ankylosis in the age range of 1-70 years who were surgically treated were assessed. The age group of 11-20 years presented with higher incidence of TMJ ankylosis $(36.36 \%)$ followed by the age group of 1-10 years $(22.73 \%$ ) [Figure 8]. Males showed higher incidence of TMJ ankylosis with $63.64 \%$ than female participants $(36.36 \%)$ [Figure 9]. The distribution of patients with Temporomandibular joint ankylosis based on Sawhney's classification showed that Sawhney's type II (45.45\%) was predominantly present among the TMJ Ankylosis patients followed by Sawhney's type I (31.82\%). Sawhney's classification type III was present in $13.64 \%$ of patients and Sawhney's type IV was seen in $9.09 \%$ of patients [Figure 10].

Right side of TMJ showed higher incidence of TMJ ankylosis $(45.45 \%)$ followed by the left side $(36.36 \%) .18 .18 \%$ of patients exhibited bilateral involvement (right and left sides) of TMJ ankylosis [Figure 11]. Interpositional arthroplasty was the predominant surgical treatment done for TMJ ankylosis patients $(86.36 \%)$. Discopexy with high condylar shaving $(9.09 \%)$ and condylectomy with total TMJ replacement $(4.55 \%)$ are the other surgical mo-

Figure 4. Bar graph showing distribution of the type of surgical treatment done for temporomandibular joint disorders.

$\mathrm{X}$ axis represents the type of surgical treatment and $\mathrm{Y}$ axis represents the number of patients undergoing surgical treatment for TMD. $41.38 \%$ of patients were treated by High condylectomy and discopexy and $27.59 \%$ of patients with only high condylectomy.

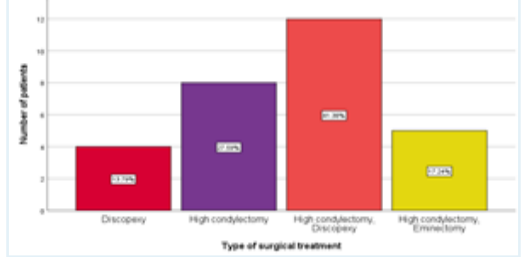

Figure 5. Bar graph depicting the association between age of the participants and the type of surgical treatment done for TMD.

$\mathrm{X}$ axis represents the age of the patients and $\mathrm{Y}$ axis represents the number of patients undergoing different surgical modalities.(Pearson's Chi square was done with $\mathrm{p}=$ $0.01(<0.05)$, hence statistically significant). Green bar represents high condylectomy and discopexy procedure done predominantly $(24.14 \%)$ in the age group of $31-40$ years. Red bar represents high condylectomy procedure done predominantly $(13.79 \%)$ in the age group of 21-30 years. Orange bars represent high condylectomy and eminectomy procedure done predominantly in 51-60 years age group (17.24\%). Blue bar represents discopexy procedure done predominantly in 10-20 years age group $(13.79 \%)$.Thus, the association between age of the participants and the type of surgical treatment done for TMD was statistically significant.

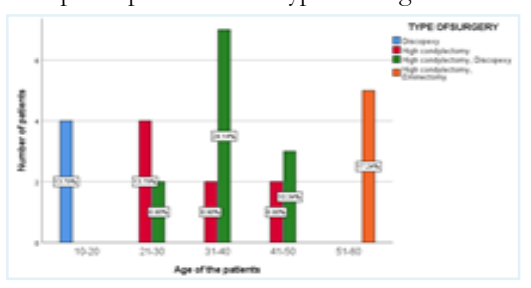

Figure 6. Bar graph depicting the association between gender of the participants and the type of surgical treatment done for TMD. $\mathrm{X}$ axis represents the gender of the patients and $\mathrm{Y}$ axis represents the number of patients undergoing different surgical modalities. (Pearson's Chi square was done with $\mathrm{p}=0.044(<0.05)$, hence statistically significant).Green bars represent high condylectomy and discopexy procedure which was done predominantly $(27.59 \%)$ in male participants. Orange bars represent high condylectomy and eminectomy procedure which was done predominantly (17.24\%) in female participants. Blue bars represent discopexy procedure which was done only in male patients $(13.79 \%)$. Thus, the association between gender of the participants and the type of surgical treatment done for TMD was statistically significant.

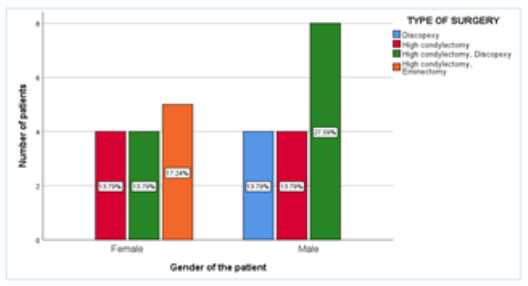


Figure 7. Bar graph depicting the association between the type of temporomandibular joint disorder and the type of surgical treatment done for TMD. $\mathrm{X}$ axis represents the type of temporomandibular joint disorder and $\mathrm{Y}$ axis represents the number of patients undergoing different surgical modalities. (Pearson's Chi square was done with $\mathrm{p}=0.032(<0.05)$, hence statistically significant).High condylectomy with discopexy was the predominant procedure done $(27.59 \%)$ for TMJ disc displacement without reduction, High condylectomy was predominantly done (13.79\%) for TMJ disc displacement with reduction, and High condylectomy with eminectomy was predominantly done (17.24\%) for TMJ subluxation/dislocation. Thus, the association between the type of temporomandibular joint disorder and the type of surgical treatment done for TMD was statistically significant.

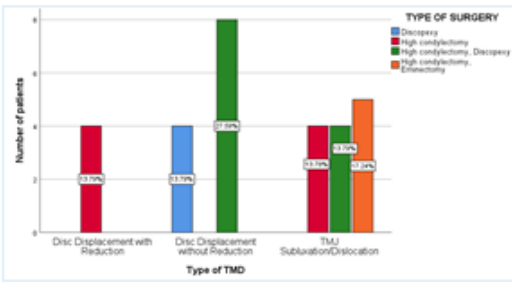

Figure 8. Bar graph showing age distribution of the patients with Temporomandibular joint ankylosis.

$\mathrm{X}$ axis represents the age group of the patients and $\mathrm{Y}$ axis represents the number of patients with TMJ ankylosis. The age group 11-20 years presented with higher incidence of TMJ ankylosis (36.36\%) followed by the age group of $1-10$ years $(22.73 \%)$.

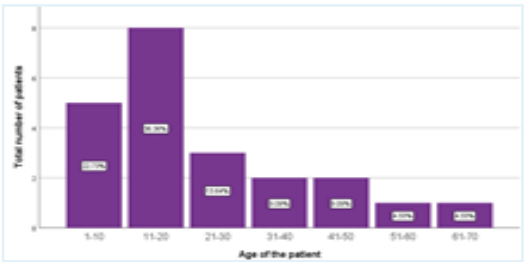

Figure 9. Bar graph showing gender distribution of the patients with Temporomandibular joint ankylosis.

$\mathrm{X}$ axis represents the gender of the patients and $\mathrm{Y}$ axis represents the number of patients with TMJ ankylosis. Males showed more incidence of TMJ ankylosis with $63.64 \%$

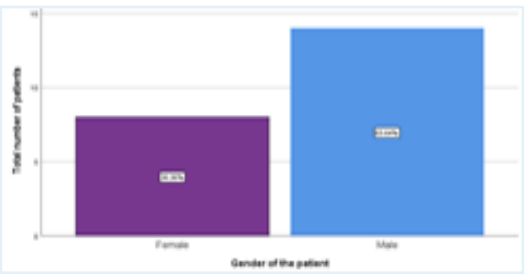

Figure 10. Bar graph showing distribution of patients with Temporomandibular joint ankylosis based on Sawhney's classification.

$\mathrm{X}$ axis represents the Sawhney's classification of TMJ ankylosis and Y axis represents the number of patients with TMJ Ankylosis. Sawhney's type II (45.45\%) was predominantly present among the TMJ Ankylosis patients followed by Sawhney's type I (31.82\%).Sawhney's classification type III was present in $13.64 \%$ of patients and Sawhney's type IV was seen in $9.09 \%$ of patients.

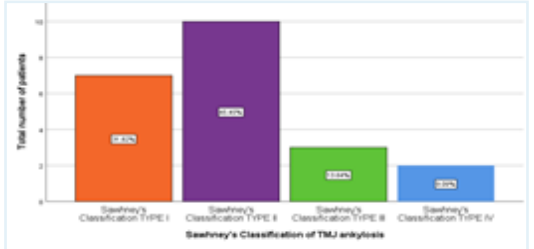

Figure 11. Bar graph showing distribution of site involved in Temporomandibular joint ankylosis patients.

$\mathrm{X}$ axis represents the site involved in TMJ ankylosis and $\mathrm{Y}$ axis represents the number of patients with TMJ Ankylosis. Right side of TMJ showed higher incidence of TMJ ankylosis (45.45\%) followed by the left side $(36.36 \%)$

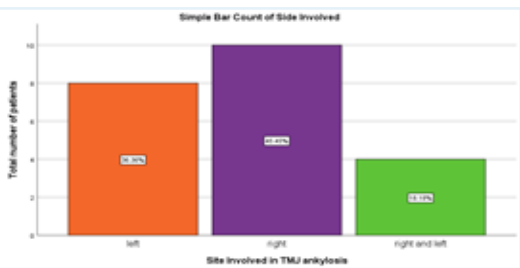

Figure 12. Bar graph showing distribution of surgical treatment modalities to treat patients with Temporomandibular joint ankylosis. $\mathrm{X}$ axis represents the surgical procedure done for treating TMJ ankylosis and $\mathrm{Y}$ axis represents the number of patients with TMJ ankylosis. Interpositional arthroplasty was the predominant surgical treatment done for TMJ ankylosis patients $(86.36 \%)$.

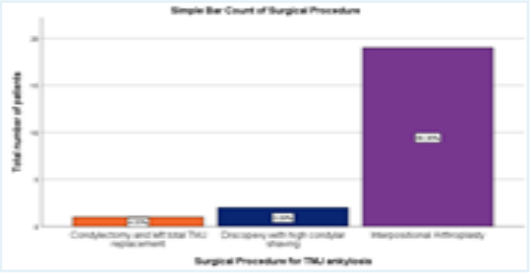


Figure 13. Bar graph showing distribution of type of interpositional material used for treating patients with Temporomandibular joint ankylosis. $\mathrm{X}$ axis represents the type of interpositional material used and $\mathrm{Y}$ axis represents the number of patients with TMJ Ankylosis. Dermis fat graft was the commonly used interpositional material $(63.64 \%)$ in the management of TMJ ankylosis.

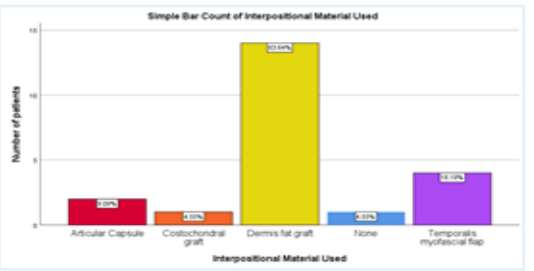

Figure 14. Bar graph depicting the association between age of the participants and Sawhney's classification of TMJ ankylosis.

$\mathrm{X}$ axis represents the age of the patients and Y axis represents Sawhney's classification of TMJ ankylosis. (Pearson's Chi square was done with $\mathrm{p}=0.010(<0.05)$, hence statistically significant).Sawhney's TYPE II is seen more in the age group 11-20 years with $22.73 \%$ followed by Sawhney's TYPE I in the age group $1-10$ years (13.64\%). Thus, the association between age of the participants and Sawhney's classification of TMJ ankylosis was statistically significant.

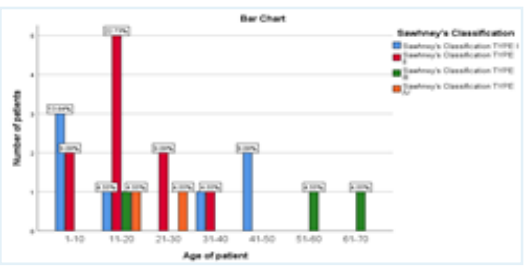

Figure 15. Bar graph depicting the association between gender of the participants and Sawhney's classification of TMJ ankylosis.

$\mathrm{X}$ axis represents the gender of the patients and $\mathrm{Y}$ axis represents Sawhney's classification of TMJ ankylosis. (Pearson's Chi square was done with $\mathrm{p}=0.044(<0.05)$, hence statistically significant). Sawhney's TYPE II was present predominantly in females with $27.27 \% \%$ and Sawhney's TYPE I occurred in males with $27.27 \%$ as well. Thus, the association between gender of the participants and Sawhney's classification of TMJ ankylosis was statistically significant.

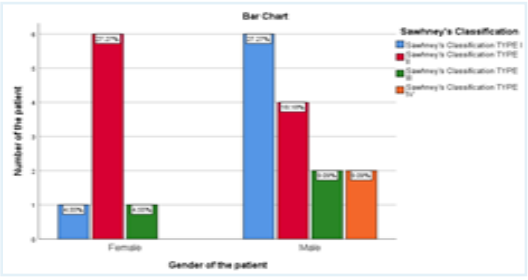

Figure 16. Bar graph depicting the association between site involved in TMJ Ankylosis and Sawhney's classification of TMJ ankylosis. $\mathrm{X}$ axis represents the site involved in TMJ ankylosis patients and $\mathrm{Y}$ axis represents Sawhney's classification of TMJ ankylosis. (Pearson's Chi square was done with $\mathrm{p}=$ $0.01(<0.05)$, hence statistically significant). Sawhney's TYPE II was present predominantly in the right side of the TMJ with $27.27 \%$ and both Sawhney's type I and II equally occurred in the left side of the TMJ (13.64\%). Thus, the association between site involved in TMJ Ankylosis and Sawhney's classification of TMJ ankylosis was statistically significant.

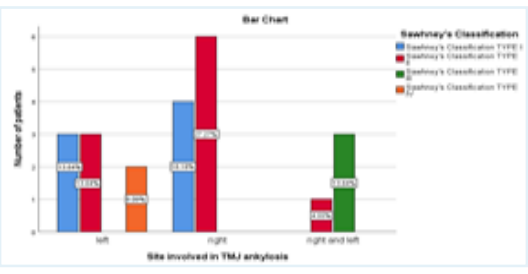

dalities employed for TMJ ankylosis patients [Figure 12]. Figure 13 shows the distribution of the type of inter-positional material used in patients with Temporomandibular joint ankylosis. Dermis fat graft was commonly used in $63.64 \%$ patients followed by temporalis myofascial flap (18.18\%), articular capsule $(9.09 \%)$, costochondral graft $(4.55 \%)$ and nil grafts in $4.55 \%$ of participants.

The association between the age of the patients and Sawhney's classification of TMJ ankylosis was evaluated and the results were statistically significant (Pearson's Chi square was done with $\mathrm{p}=$ $0.010(<0.05)$, hence statistically significant). Sawhney's TYPE II is seen more in the age group $11-20$ years with $22.73 \%$ followed by Sawhney's TYPE I in the age group 1-10 years (13.64\%). Thus, the association between age of the participants and Sawhney's classification of TMJ ankylosis was statistically significant [Figure 14].

The association between gender of the participants and Sawhney's classification of TMJ ankylosis was evaluated and the results were statistically significant (Pearson's Chi square was done with $\mathrm{p}$ $=0.044(<0.05)$, hence statistically significant). Sawhney's TYPE
II was present predominantly in females with $27.27 \% \%$ and Sawhney's TYPE I occurred in males with $27.27 \%$ as well. Thus, the association between gender of the participants and Sawhney's classification of TMJ ankylosis was statistically significant [Figure $15]$.

The association between site involved in TMJ Ankylosis and Sawhney's classification of TMJ ankylosis was evaluated and the results were statistically significant (Pearson's Chi square was done with $\mathrm{p}=0.01(<0.05)$, hence statistically significant). Sawhney's TYPE II was present predominantly in the right side of the TMJ with $27.27 \%$ and both Sawhney's type I and II equally occurred in the left side of the TMJ (13.64\%). Thus, the association between site involved in TMJ Ankylosis and Sawhney's classification of TMJ ankylosis was statistically significant [Figure 16].

Quality of life after surgery in these patients was assessed using pain scale - visual analogue scale [VAS], and mouth opening [both preoperative and post-operative] periodically and there was a good improvement in form and function. Evaluation scores at 1- month post-operative was recorded and compared with pre- 
operative levels which showed statistically significant differences and these were maintained during the subsequent periodic visits at 2nd, 3rd, 6th, 9th and 12th months.

\section{One-month post-operative review}

The mean mouth opening in 30 patients with TMD before surgical therapy was $36.80 \mathrm{~mm}$ and after surgical therapy was 41.50 $\mathrm{mm}$. This increase (improvement) in mean mouth opening of $4.70 \mathrm{~mm}$ after surgical therapy was found to be statistically significant $[\mathrm{p}=0.001$, paired ' $\mathrm{t}$ ' test] [Figure 17]. The mean visual analogue scale [VAS] pain score in 30 patients with TMD before surgical therapy was 6.77 and after surgical therapy was 1.10 . This reduction (improvement) in mean pain score of 5.67 after surgical therapy was found to be statistically significant $[\mathrm{p}=0.001$, paired ' $\mathrm{t}$ ' test] [Figure 18].

The mean mouth opening in 23 patients with TMJ Ankylosis before surgical therapy was $33 \mathrm{~mm}$ and after surgical therapy was
$40.78 \mathrm{~mm}$. This increase (improvement) in mean mouth opening of $7.78 \mathrm{~mm}$ after surgical therapy was found to be statistically significant [ $\mathrm{p}=0.001$, paired ' $\mathrm{t}$ ' test] [Figure 19]. The mean visual analogue scale [VAS] pain score in 23 patients with TMJ Ankylosis before surgical therapy was 6 and after surgical therapy was 1.78 . This reduction (improvement) in mean pain score of 4.23 after surgical therapy was found to be statistically significant $[p=0.001$, paired 't' test] [Figure 20].

\section{Discussion}

According to our study, Male patients predominantly underwent surgery for TMD especially in the age group of 31-40 years. Also, surgical treatment such as high condylectomy and discopexy was seen to be successful in the treatment of TMD. Similarly in a Randomized controlled trial carried out by Vos et al, it was seen that arthrocentesis was more effective compared to conservative treatment as initial treatment with regard to temporomandibu-

Figure 17. Bar graph depicting Mean maximal mouth opening ( \pm SEM) in patients with disc-condyle disorders [TMD] who were surgically treated. Preoperative data are shown in blue and responses at the one-month postoperative test in green. There was statistically significant increase in maximal mouth opening one-month postoperatively as assessed by maximal interincisal distance, compared with preoperative levels. (Maximal mouth opening: $\mathrm{p}=0.001$; paired Student's $\mathrm{t}$ test). Post-op = one-month postoperative test; pre-op = pre- operatively; SEM = standard error of the mean.

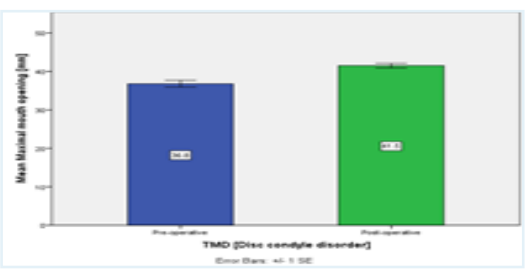

Figure 18.Bar graph depicting Mean VAS scores ( \pm SEM) for the level of pain in patients with disc-condyle disorders [TMD] who were surgically treated. Patients scored their symptoms on a $10 \mathrm{~mm}$ scale from $0=$ an absence of symptoms to $10=$ worst imaginable (pain and discomfort). Preoperative data are shown in blue and responses at the one-month postoperative test in green. There were statistically significant reductions in symptoms one-month postoperatively as assessed by VAS, compared with preoperative levels for pain. (Pain: $\mathrm{p}=0.001$; paired Student's $\mathrm{t}$ test). Post-op $=$ one-month postoperative test; pre-op $=$ pre- operatively; SEM $=$ standard error of the mean; VAS = visual analogue scale.

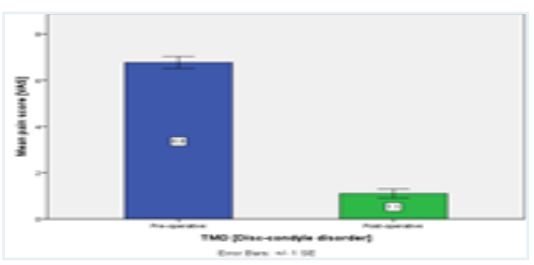

Figure 19.Bar graph depicting Mean maximal mouth opening in mm ( \pm SEM) in Ankylosis [TMD] who were surgically treated.

Preoperative data are shown in blue and responses at the one-month postoperative test in green. There was statistically significant increase in maximal mouth opening onemonth postoperatively as assessed by maximal interincisal distance compared with preoperative levels (Maximal mouth opening: $\mathrm{p}=0.001$; paired Student's $\mathrm{t}$ test). Post-op $=$ one-month postoperative test; pre-op = pre- operatively; SEM = standard error of the mean.

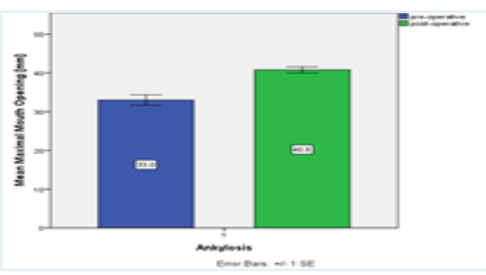

Figure 20.Bar graph depicting Mean VAS scores ( \pm SEM) for the level of pain in patients with Ankylosis [TMD] who were surgically treated.

Patients scored their symptoms on a $10 \mathrm{~mm}$ scale from $0=$ an absence of symptoms to $10=$ worst imaginable (pain and discomfort). Preoperative data are shown in blue and responses at the one-month postoperative test in green. There were statistically significant reductions in symptoms one-month postoperatively as assessed by VAS, compared with preoperative levels for pain. (Pain: $\mathrm{p}=0.001$; paired Student's t test). Post-op $=$ one-month postoperative test; pre-op $=$ pre- operatively; SEM $=$ standard error of the mean; VAS = visual analogue scale.

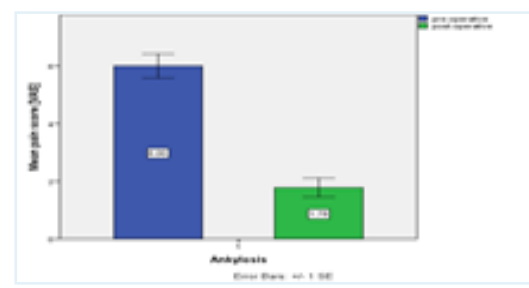


lar joint pain and mandibular movement. They showed that arthrocentesis reduces pain and functional impairment more rapidly compared to conservative treatment [30]. If the minimally invasive procedures like TMJ arthrocentesis are not successful in treating TMD's, then surgical modality such as high condylectomy with discopexy are very effective. It is very useful for TMD's, especially in managing TMJ disc displacement without reduction/ subluxation. According to our study, high condylectomy with eminectomy procedure is a useful surgical modality for treating TMJ dislocation/subluxationand it was performed mostly for females. Also, only high condylectomy was done for treating patients with disc displacement with reduction.

In our study, the age group between 1-20 years showed more incidences of TMJ ankylosis with male preponderance. Similarly, in a study by Akhtar et al., [31] it is stated that TMJ ankylosis was commonly seen in children and young adults and most of the patients were in the 11-15 years' age group. Various hospital-based studies showed that the most prevalent age group presenting with TMJ ankylosis is $6-10$ year and $11-20$ years $[31,32]$. In our study, males showed more incidence of TMJ ankylosis which is similar to the cases reported in the studies by Vasconcelos et al., [33] and Garcia et al [34]. However, in a study by Erol et al., [35] a significant proportion of females than males exhibited TMJ ankylosis.

In our study, unilateral TMJ ankylosis (right or left site involvement) was more prevalent with a preponderance for right side TMJ. However, in a study by Gupta et al, bilateral cases were more than the unilateral ones [36]. In our study, according to Sawhney's classification, type II was predominantly present among the TMJ Ankylosis patients especially on the right side and inter-positional arthroplasty with dermis graft was the commonly performed surgical modality for treating TMJ Ankylosis. Inter-positional arthroplasty $(86 \%)$ was the preferred choice of treating TMJ ankylosis patients in our study population and it produced stable and excellent results which is similar to that reported in several other studies. In $5 \%$ of patients, condylectomy and total TMJ prosthetic replacement was done due to the presence of TMJ pathology.

The insertion of soft tissue between the condyle and glenoid fossa is essential for the reduction of recurrence [37]. In our study $18 \%$ of patients received temporalis myofascial flaps as an interpositional material with favourable results, which is similar to a study that showed patients with ankylosis type III who received temporalis myofascial flaps as inter-positional material had longterm stable therapeutic effects [38]]. In our study dermis fat graft was used as an inter-positional material in $64 \%$ of patients with TMJ ankylosis and it was successful in preventing recurrence and improving form and function of the TMJ over a longer period of time. Similar to our study, Mehrotra et al., [39] and Thangavelu et al [40] in their studies confirmed the long-term survival and success rate of dermal fat interposition arthroplasty for management of TMJ ankylosispatients. Dimitroulis et al., [41, 42] also found that free fat insertion can reduce the recurrence of TMJ ankylosis through clinical and animal experiments.

With a rich case bank established in our institution over the last decade, we have been able to publish extensively in this domain [43-52]. Drawbacks of this study consist of limited population being studied and inclusion of a smaller sample size. Future scope of the study is that a larger sample from other parts of the population can be assessed over a longer period of time.

\section{Conclusion}

It can be concluded from our study that TMJ disorders are now becoming common among the younger population especially the males. High condylectomy and discopexy was the commonest procedure done successfully for patients with disc-condyle disorders. High condylectomy with eminectomy was predominantly done for TMJ subluxation/dislocation. Interpositional arthroplasty with dermis fat graft was the predominant procedure done for treating TMJ ankylosis patients and the results were stable. This study highlights the role of various surgical treatment modalities to treat TMD and the high-risk group for developing temporomandibular joint disorders. Early diagnosis and adequate surgical intervention would minimize the deformity in TMJ ankylosis patients. Thus, surgical treatment modalities can be advised for indicated cases in TMDs, where conservative treatment modalities cannot offer proper cure for the patients. In certain cases of TMD, surgical modality may be the first and only option for the management. When performed at prompt time, surgical modalities can improve form, function and aesthetics of the patients thereby improving the overall outcome and general and oral health related quality of life of the patients. There has been a recent increase in awareness towards TMJ disorders among the population, however the attitude of TMD patients towards undergoing therapy is still sparse and dental practitioners should motivate their patients. It can be understood that a multidisciplinary team approach to TMD management is essential in the fundamental care of all TMD patients.

\section{References}

[1]. Dimitroulis G. Temporomandibular disorders: a clinical update. Bmj. 1998 Jul 18;317(7152):190-4.

[2]. Schiffman E, Ohrbach R, Truelove E, Look J, Anderson G, Goulet JP, et al. Diagnostic Criteria for Temporomandibular Disorders (DC/TMD) for Clinical and Research Applications: recommendations of the International RDC/TMD Consortium Network and Orofacial Pain Special Interest Group. J Oral Facial Pain Headache. 2014 Winter;28(1):6-27.Pubmed PMID: 24482784

[3]. Bernhardt O, Gesch D, Schwahn C, Mack F, Meyer G, John U, et al. Epidemiological evaluation of the multifactorial aetiology of abfractions. J Oral Rehabil. 2006 Jan;33(1):17-25.Pubmed PMID: 16409512.

[4]. Liu F, Steinkeler A. Epidemiology, diagnosis, and treatment of temporomandibular disorders. Dental Clinics. 2013 Jul 1;57(3):465-79.

[5]. Guarda-Nardini L, Pavan C, Arveda N, Ferronato G, Manfredini D. Psychometric features of temporomandibular disorders patients in relation to pain diffusion, location, intensity and duration. J Oral Rehabil. 2012 Oct;39(10):737-43.Pubmed PMID: 22631608.

[6]. Bono AE, Learreta JA, Rodriguez G, Marcos JC. Stomatognathic system involvement in rheumatoid arthritis patients.cranio. 2014 Jan 1;32(1):31-7.

[7]. Dimitroulis G. Temporomandibular joint surgery: what does it mean to the dental practitioner?. Aust. Dent. J. 2011 Sep;56(3):257-64.

[8]. Dimitroulis G. Management of temporomandibular joint disorders: A surgeon's perspective. Aust. Dent. J. 2018 Mar;63:S79-90.

[9]. Guarda-Nardini L, De Almeida AM, Manfredini D. Arthrocentesis of the Temporomandibular Joint: Systematic Review and Clinical Implications of Research Findings. J Oral Facial Pain Headache. 2021 Winter;35(1):17-29. Pubmed PMID: 33730123.

[10]. Forssell H, Kotiranta U, Kauko T, Suvinen T. Explanatory Models of Illness and Treatment Goals in Temporomandibular Disorder Pain Patients Reporting Different Levels of Pain-Related Disability. J Oral Facial Pain Headache. 2016 Winter;30(1):14-20.Pubmed PMID: 26817028.

[11]. Dolwick MF, Dimitroulis G. Is there a role for temporomandibular joint surgery?. Br J Oral Maxillofac Surg. 1994 Oct 1;32(5):307-13.

[12]. Larheim TA. Role of magnetic resonance imaging in the clinical diagnosis of the temporomandibular joint. Cells Tissues Organs. 2005;180(1):6-21.

[13]. Vogl TJ, Lauer HC, Lehnert T, Naguib NN, Ottl P, Filmann N, et al. The value of MRI in patients with temporomandibular joint dysfunction: correlation of MRI and clinical findings. Eur. J. Radiol. 2016 Apr 1;85(4):714-9. 
[14]. Mercuri LG. Temporomandibular joint total joint replacement-TMJ TJR. Switzerland: Springer Int Pub. 2016.

[15]. Holmlund A, Hellsing G. Arthroscopy of the temporomandibular joint: occurrence and location of osteoarthrosis and synovitis in a patient material. Int J Oral Maxillofac Surg. 1988 Feb;17(1):36-40.Pubmed PMID: 3127488.

[16]. Montgomery MT, Gordon SM, Van Sickels JE, Harms SE. Changes in signs and symptoms following temporomandibular joint disc repositioning surgery. J Oral Maxillofac Surg. 1992 Apr 1;50(4):320-8.

[17]. Bouloux G, Koslin MG, Ness G, Shafer D. Temporomandibular joint surgery. J Oral Maxillofac Surg. 2017 Aug 1;75(8):e195-223.

[18]. Dimitroulis G. The role of surgery in the management of disorders of the Temporomandibular Joint: a critical review of the literature. Part 1. Int J Oral Maxillofac Surg. 2005 Mar;34(2):107-13.Pubmed PMID: 15695036.

[19]. Dimitroulis G. The role of surgery in the management of disorders of the temporomandibular joint: a critical review of the literature: Part 2. Int J Oral Maxillofac Surg. 2005 May 1;34(3):231-7.

[20]. Tocaciu S, McCullough MJ, Dimitroulis G. Surgical management of recurrent TMJ dislocation-a systematic review. Oral Maxillofac Surg. 2019 Mar;23(1):35-45.Pubmed PMID: 30729355.

[21]. Akinbami BO. Evaluation of the mechanism and principles of management of temporomandibular joint dislocation. Systematic review of literature and a proposed new classification of temporomandibular joint dislocation. Head Face Med. 2011 Jun 15;7:1-9.Pubmed PMID: 21676208.

[22]. Melo AR, Júnior EP, Santos LD, Vasconcelos BD. Recurrent dislocation: scientific evidence and management following a systematic review. Int. J. Oral Maxillofac. Surg. 2017 Jul 1;46(7):851-6.

[23]. Prechel U, Ottl P, Ahlers OM, Neff A. The treatment of temporomandibular joint dislocation: A systematic review. Dtsch Arztebl Int. 2018 Feb;115(5):59.

[24]. Chidzonga MM. Temporomandibular joint ankylosis: review of thirty-two cases. Br J Oral Maxillofac Surg. 1999 Apr 1;37(2):123-6.

[25]. Sawhney CP. Bony ankylosis of the temporomandibular joint: follow-up of 70 patients treated with arthroplasty and acrylic spacer interposition. Plast Reconstr Surg. 1986 Jan;77(1):29-40.Pubmed PMID: 3941847.

[26]. Su-Gwan K. Treatment of temporomandibular joint ankylosis with temporalis muscle and fascia flap. Int. J. Oral Maxillofac. Surg. 2001 Jun 1;30(3):189-93.

[27]. Kaban LB, Perrott DH, Fisher K. A protocol for management of temporomandibular joint ankylosis. J Oral Maxillofac Surg. 1990 Nov $1 ; 48(11): 1145-51$.

[28]. Kaban LB, Bouchard C, Troulis MJ. A protocol for management of temporomandibular joint ankylosis in children. J Oral Maxillofac Surg. 2009 Sep $1 ; 67(9): 1966-78$

[29]. Lin X, Li HY, Xie QT, Zhang T, Huang XP, Zhou N. Surgical treatment of type III temporomandibular joint ankylosis with a lateral arthroplasty while retaining the medially displaced condyle. Ann R Coll Surg Engl. 2019 Jul;101(6):415-421.Pubmed PMID: 31155887.

[30]. Vos LM, Slater JH, Stegenga B. Arthrocentesis as initial treatment for temporomandibular joint arthropathy: a randomized controlled trial. J Craniomaxillofac Surg. 2014 Jul 1;42(5):e134-9.

[31]. Akhtar MU, Shah AA, Abbas I. Treatment of unilateral Temporomandibular joint ankylosis with silastic interpositional Arthroplasty. Ann. King Edw. Med. Univ. 2005;11(4).

[32]. Qudah MA, Qudeimat MA, Al-Maaita J. Treatment of TMJ ankylosis in Jordanian children - a comparison of two surgical techniques. J Craniomaxillofac Surg. 2005 Feb;33(1):30-6.Pubmed PMID: 15694147.

[33]. do Egito Vasconcelos BC, Bessa-Nogueira RV, Cypriano RV. Treatment of temporomandibular joint ankylosis by gap arthroplasty. Med Oral Patol Oral Cir Bucal. 2006 Jan 1;11:E66-9.

[34]. Garcia-Aparicio L, Parri FJ, Sancho MA, Sarget R, Morales L. Temporomandibular joint ankylosis (TMA) in children. Cirugia pediatrica: organo oficial de la Sociedad Espanola de Cirugia Pediatrica. 2000 Apr 1;13(2):62-3.

[35]. Erol B, Tanrikulu R, Görgün B. A clinical study on ankylosis of the temporomandibular joint. J Craniomaxillofac Surg. 2006 Mar 1;34(2):100-6.

[36]. Gupta VK, Mehrotra D, Malhotra S, Kumar S, Agarwal GG, Pal US. An epidemiological study of temporomandibular joint ankylosis. Natl. j. maxillofac. surg. 2012 Jan;3(1):25.

[37]. He D, Yang C, Chen M, Yang X, Li L, Jiang Q. Surgical treatment of traumatic temporomandibular joint ankylosis with medially displaced residual condyle: surgical methods and long-term results. J Oral Maxillofac Surg. 2011 Sep 1;69(9):2412-8.

[38]. Singh V, Bhagol A, Dhingra R, Kumar P, Sharma N, Singhal R. Management of temporomandibular joint ankylosis type III: lateral arthroplasty as a treatment of choice. Int J Oral Maxillofac Surg. 2014 Apr;43(4):460-4. Pubmed PMID: 24100155.

[39]. Mehrotra D, Pradhan R, Mohammad S, Kumar S. Complications associated with different surgical modalities for management of temporomandibular ankylosis in a series of 791 cases. Asian Journal of Oral and Maxillofacial Surgery. 2011 Aug 1;23(3):122-7.

[40]. Thangavelu A, Thiruneelakandan S, Prasath CH, Chatterjee D. Fate of free fat dermis graft in TMJ interpositional gap arthroplasty: a long term MRI study. J Oral Maxillofac Surg. 2015 Jun;14(2):321-6.

[41]. Dimitroulis G, Slavin J, Morrison W. Histological fate of abdominal dermisfat grafts implanted in the temporomandibular joint of the rabbit following condylectomy. Int J Oral Maxillofac Surg. 2011 Feb;40(2):177-83.Pubmed PMID: 21050720.

[42]. Dimitroulis G. The interpositional dermis-fat graft in the management of temporomandibular joint ankylosis. Int. J. Oral Maxillofac. Surg.. 2004 Dec 1;33(8):755-60.

[43]. MP SK. Knowledge, Attitude and practices regarding needlestick injuries among dental students. Asian J Pharm Clin Res. 2016;9(4):312-5.

[44]. SK M. Knowledge, attitude, and practices regarding infection control among undergraduate dental students. Asian J Pharm Clin Res. 2016;9(1):220-4.

[45]. Santhosh KM, Harshini AK. Knowledge and awareness about oral cancer among undergraduate dental students. Asian J Pharm Clin Res. 2016;9(4): 165-7.

[46]. Gayathri MM. Knowledge and awareness among patients about dental implants. J. Pharm. Sci. Res. 2016 May 1;8(5):351.

[47]. Vijayalakshmi B, Kumar MS. Knowledge of students about Local anaesthetics used during oral surgical procedures. J. Pharm. Sci. Res. 2015 Nov $1 ; 7(11): 1011$.

[48]. Gayathri MM. Knowledge, Awareness and Attitude among dental students about hepatitis B infection. J. Pharm. Sci. Res. 2016 Mar 1;8(3):168.

[49]. Ahamed A, Kumar MS. Knowledge, attitude and perceived confidence in handling medical emergencies among dental students. J. Pharm. Sci. Res. 2016 Jul 1;8(7):645.

[50]. Kumar S. Knowledge, attitude and practices of dental students toward dental management of patients on antiplatelet therapy. Asian J Pharm Clin Res. 2016;9(30):270-6

[51]. Mp SK. Local hemostatic agents in the management of bleeding in oral surgery. Asian J Pharm Clin Res. 2016;9(3):35-41.

[52]. Kumar MP. Newer methods of extraction of teeth. Int J Pharm Bio Sci. 2015;6(3):679-85. 